\title{
Paraneoplastic Cerebellar Ataxia Can Affect Prognosis in High-Grade Serous Ovarian Cancer: A Case Report
}

\author{
Aikaterini Liapi Apostolos Sarivalasis \\ Center of Gynecological Tumors, Oncology Department, CHUV - Centre Hospitalier \\ Universitaire Vaudois, Lausanne, Switzerland
}

Keywords

Ovarian cancer $\cdot$ Paraneoplastic syndrome $\cdot$ Prognosis

\begin{abstract}
The reported case is a 61-year-old woman, admitted for gradual onset of gait disturbances and dysphonia. The serum immunological panel revealed anti-Yo autoantibodies, suggestive of a paraneoplastic syndrome (PNS). A PET-CT revealed a suspicious left ovarian mass with retroperitoneal nodal involvement, and the histological assessment of surgical samples confirmed a FIGO IIIC high-grade serous ovarian cancer (HGSOC). Deemed inoperable at first, the patient was treated by carboplatin and paclitaxel chemotherapy, after which she refused surgical debulking. At the end of her systemic treatment, the patient only experienced a transient improvement of the cerebellar ataxia. Despite the suboptimal oncological treatment, the patient still presents stable disease and is free of progression 7 years from her diagnosis. This case study illustrates the favorable effect of PNS occurrence on oncological outcome in a patient with advanced HGSOC. The absence of recurrence despite the presence of residual disease after the systemic treatment is unusual and could be related to the PNS.
\end{abstract}

(C) 2020 The Author(s)

Published by S. Karger AG, Basel

\section{Introduction}

Ovarian cancer (OC) is the fifth most common type of cancer in women. The majority of cases of OC are of epithelial origin ( 90\%). OC is frequently asymptomatic at initial stages. The most common symptoms include abdominal or pelvic pain, bowel changes, 


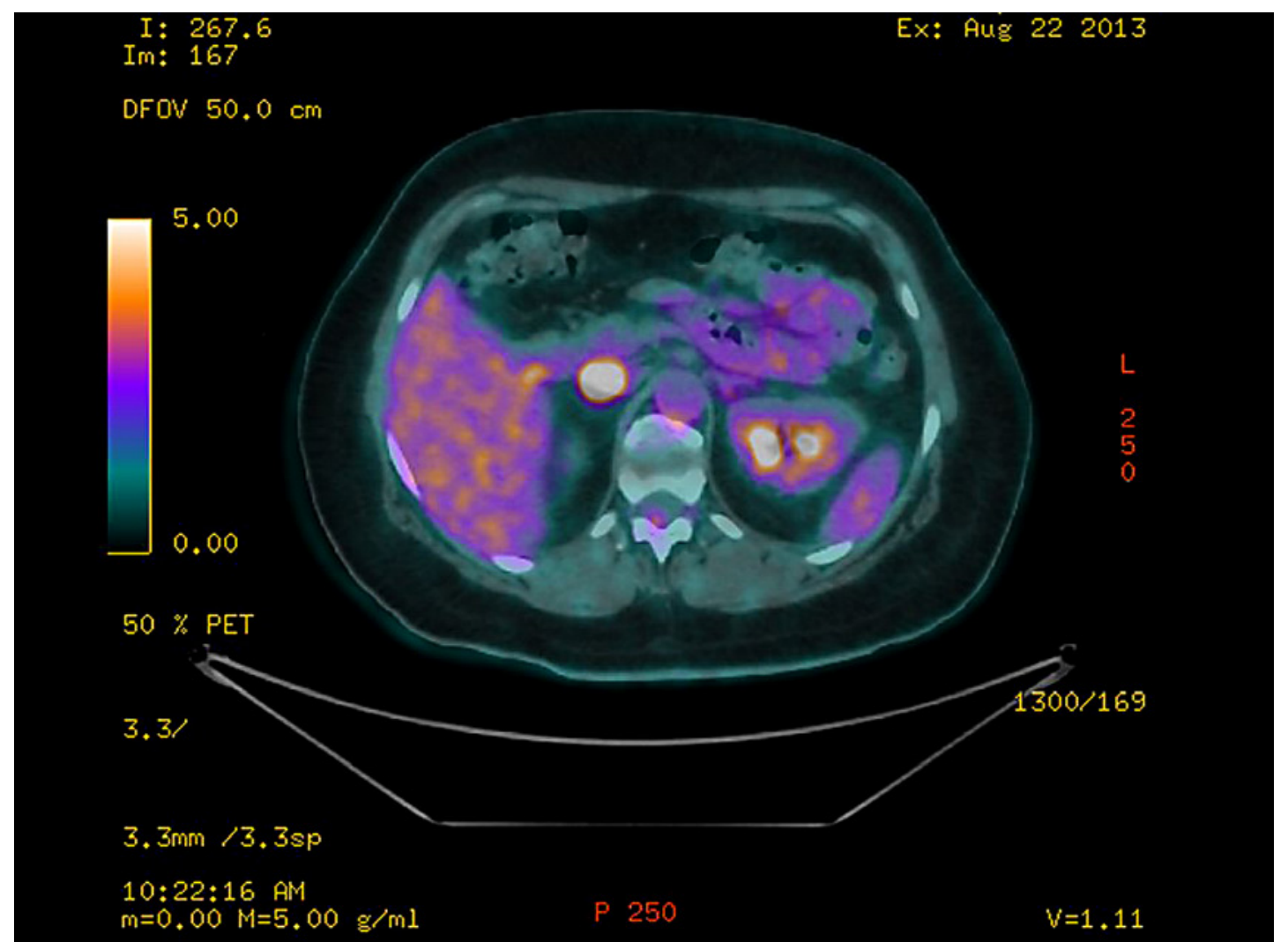

Fig. 1. Initial PET-CT. Disease extension at diagnosis.

vaginal bleeding, abdominal distension, and fatigue, and they often appear in advanced stages. Rarely, ovarian carcinomas can be related to paraneoplastic neurological syndromes. Paraneoplastic syndrome (PNS) can be either the presenting symptom or part of the clinical manifestations of the underlying cancer. When PNS is the initial presentation of OC, it can mask and delay the diagnosis of the underlying disease [1]. PNS occurrence is considered as a favorable factor for tumor prognosis and cancer treatment outcome [2]. However, the most important prognostic factor for patients with advanced OC is residual disease after debulking surgery. Therefore, a maximal debulking effort is the gold standard for the primary treatment of OC.

Since the mid-1990s, primary debulking surgery followed by platinum- and taxane-based combination chemotherapy has been the standard of care for OC treatment. The aim of the surgical treatment is complete cytoreduction of all macroscopic visible disease, since this has been shown to be associated with a significantly increased overall survival (OS) and progression-free survival $[3,4]$. The timing of cytoreductive surgery in relation to chemotherapy is debated, with accumulating data supporting neoadjuvant chemotherapy as a noninferior treatment option for patients frail or with disease too extensive to undergo primary debulking surgery [5].

We report the case of a patient presenting with a PNS as the first symptom of an underlying advanced high-grade serous ovarian cancer (HGSOC). Despite having been treated only by systemic chemotherapy, as she refused surgical debulking, the patient had a very good partial response, with persistence of only one left iliac lymphadenopathy. Her disease is still stable 7 years after the diagnosis. 


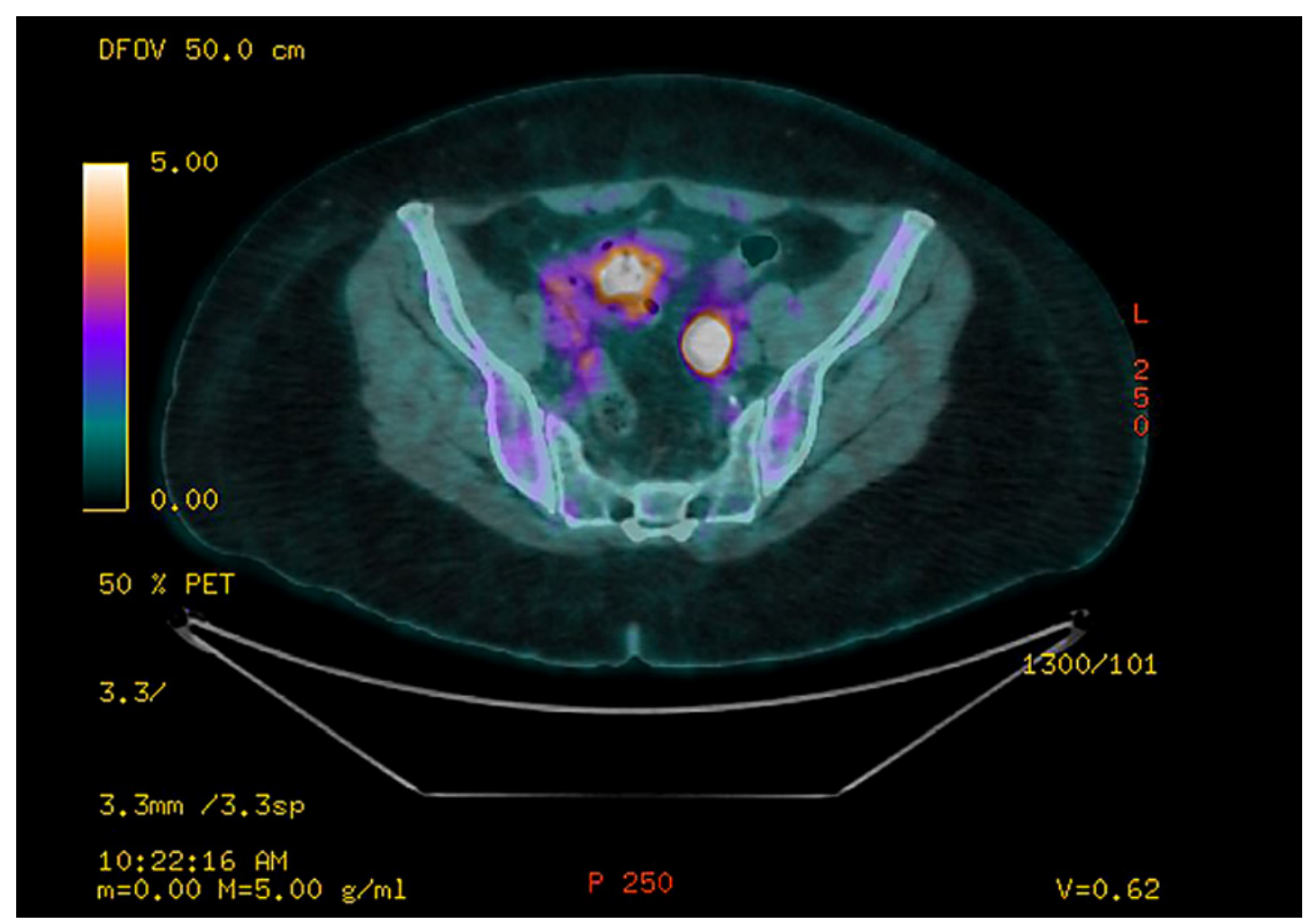

Fig. 2. Initial PET-CT. Disease extension at diagnosis.

\section{Case Report}

A previously healthy 61-year-old woman was referred to the hospital for investigation of recent onset of neurological symptoms. At admission, the patient complained of increasing gait disturbances and dysphonia, which had appeared gradually.

The neurological examination showed dysmetria, dysdiadochokinesia, dysarthria, and ataxia of stance and gait [6], and the patient was hospitalized for investigations of a rapidly progressive predominantly static and lower-limb cerebellar syndrome.

The initial radiological assessment by brain magnetic resonance imaging (MRI) showed diffuse cerebellar atrophy, predominant in the vermis. The neuropsychological examination revealed moderate executive dysfunction. Nerve conduction examination showed areflexia and the isolated absence of sensory potentials on the right superficial peroneal nerve.

Since the differential diagnosis of cerebellar ataxia is broad [7], the patient benefited from a large biological assessment, which did not reveal any abnormality of the renal or hepatic function or an inflammatory syndrome. Vitamin balance (vitamins B12, B9, and E) as well as thyroid function were also in normal range. The assay of tumor markers showed an increase in CA-125 (307 kU/L). Genetic examination for the most frequent spinocerebellar ataxias (SCA 1, 2, 3, 6, 7, and 17 genes) as well as for Friedreich's ataxia was negative. The lumbar puncture showed a discrete elevation of protein concentration without pleocytosis, but with an intrathecal synthesis of IgG.

In order to rule out a paraneoplastic neurological syndrome, a PET-CT was carried out (Fig. 1-3). It showed 3 suspicious left ovarian masses and 2 pathological lymph nodes near the portal vein and the vena cava. The diagnosis of a cerebellar degeneration of paraneo- 


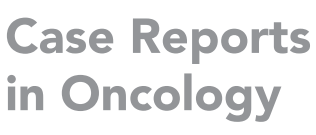

Liapi/Sarivalasis: Paraneoplastic Neurological Syndrome Combined with Ovarian Cancer

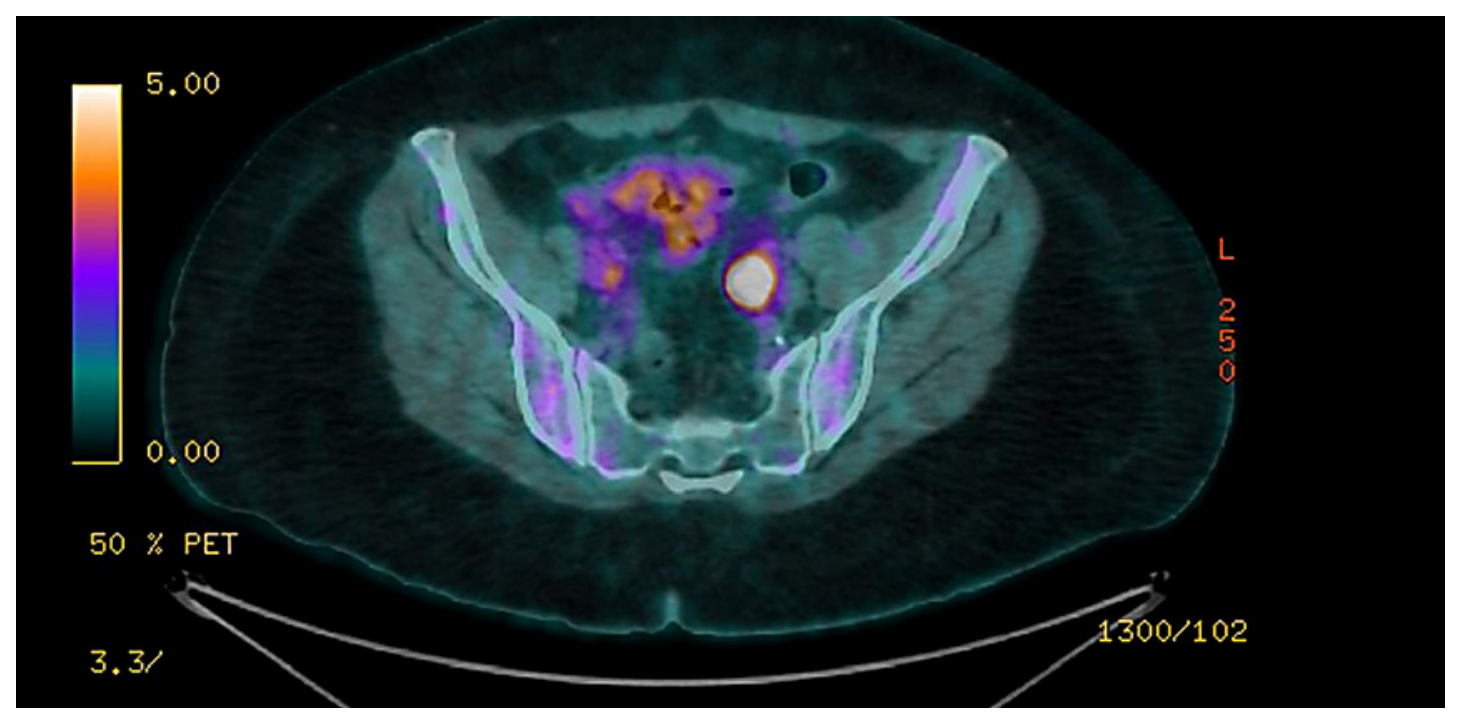

Fig. 3. Initial PET-CT. Disease extension at diagnosis.

plastic origin was thus retained. It was supported by the detection of positive anti-Yo antineuronal antibodies [1].

To confirm the tumor diagnosis, a diagnostic laparoscopy with peritoneal washing and biopsies was performed. The histopathological analysis confirmed a FIGO IIIC HGSOC. The frail condition of the patient made primary debulking surgery efforts impossible. Therefore, the patient underwent a 6-cycle, 3-weekly carboplatin treatment dosed at 6 area under the curve and paclitaxel $175 \mathrm{mg} / \mathrm{m}^{2}$ chemotherapy treatment. Beside a satisfactory clinical, biological, and radiological response, with persistence of a left iliac lymph node only, the patient, still affected by her PNS, refused surgical debulking. Based on former treatment algorithms, she received a 15 -month treatment of $7.5 \mathrm{mg} / \mathrm{kg}$ bevacizumab maintenance treatment. Her BRCA 1/2 mutational status is unknown.

The patient went on to standard follow-up by periodic radiological assessment and CA-125 dosing. Seven years after her diagnosis, the radiological assessments show the persistence and stability of the left iliac lymph node, without any sign of progression.

With respect to the paraneoplastic neurological syndrome evolution, despite a transient improvement of the cerebellar ataxia and dysarthria, the neurological impairment persists to this day. Neurorehabilitation, physiotherapy, and speech therapy did not help improve the neurological deficit of the patient. No other treatments, including corticosteroids, immunoglobulins, or plasma apheresis, were prescribed. On brain imaging, a stable, over the years diffuse, central nervous system atrophy persists.

\section{Discussion/Conclusion}

In our case report, we describe the diagnosis, treatment, and outcome of a patient suffering from a PNS as the presenting symptom of an advanced HGSOC. Although the patient had a suboptimal treatment without surgical debulking of the tumor, she experienced an unexpectedly long stable disease and is still free of progression. This patient's history confirms reported observations associating the occurrence of PNS with a favorable oncological outcome [2].

High-grade serous is the most common subtype of epithelial ovarian adenocarcinomas. Their prognosis is mostly affected by stage at diagnosis and the amount of residual tumor 
after debulking surgery. OC staging guidelines used in our patient were updated in 2014. In the absence of peritoneal disease, isolated lymph node involvement beyond the pelvis was no longer considered as stage IIIC but as stage IIIA2 disease. This update from the FIGO 2009 to the FIGO 2014 staging system reflects the observed lesser impact of lymph node involvement in the overall prognosis of the disease, especially for stage III and IV OC without bulky lymph nodes. Although currently the disease stage of our patient would be less advanced, the lack of debulking surgery, irrespective of the systemic platinum-based chemotherapy, is a wellestablished negative prognostic factor [3]. Virtually, all patients with macroscopically residual disease experience early recurrences and shorter OS [3,4]. Thus, nonsurgical treatment of epithelial OC is not considered as a standard of care and is often regarded as palliative care. Indeed, Shalowitz et al. [8] reported in 2016 that about $20 \%$ of women diagnosed with OC do not undergo surgery. These patients are aged 75 years or older and have advanced-stage disease. In this cohort, patients treated with surgery had an OS of 57 months. On the contrary, nondebulked patients had less than 12 months of OS, while patients who received no treatment at all had an OS of just 1.4 months. Although there are no prospective trials comparing surgical to nonsurgical treatment outcomes, based on the available literature, optimal surgical cytoreductive effort is the milestone of the medical care of patients with newly diagnosed ovarian epithelial carcinoma.

Therefore, the unexpectedly long survival without signs of progression of our patient is rare and worth mentioning. Neither the FIGO stage update, nor the systemic treatment could account for this exceptional outcome. Another potential factor that could favorably influence the prognosis of our patient is the presence of a BRCA1/2 mutation. Although BRCA mutationassociated OC tends to have a favorable prognosis and response to platinum treatment compared to wild type, counterparts at equal stage, nondebulked OCs do not show such a long OS with chemotherapy alone. Thus, the occurrence of the PNS seems to have positively affected the outcome of our patient.

Neurological manifestations seen in cancer patients are mostly the consequence of direct tumor invasion of the nervous system or presence of metastases. PNS are rare conditions, present in 1-3\% of oncological patients and associated with a broad variety of tumors. They are a heterogeneous set of cancer-related neurological diseases and may involve any part of the nervous system, but they are not caused by a direct malignant tumor infiltration [9]. PNSrelated neurological symptoms can be the presenting symptoms in up to $70 \%$ of patients [1, 9], and so they can help in the early diagnosis of the tumor, permitting the instauration of treatment at earlier stages [10]. As a result, cancer prognosis of patients presenting with PNS is usually better, although their global survival may be poor due to severe neurological morbidity [11].

The most common PNS associated with ovarian tumors are subacute cerebellar degeneration and subacute sensory neuropathy $[1,9,10,12]$. Paraneoplastic cerebellar degeneration is believed to be due to an autoimmune reaction, targeted against components of the central nervous system, mostly Purkinje cells. It can result in rapid pan-cerebellar dysfunction, it can manifest several months before the presentation of neoplasms, and it is characterized by subacute onset of truncal and limb ataxia, dysarthria, and nystagmus. The ataxia can be asymmetrical at the initial presentation but usually becomes symmetrical during the disease evolution. Cerebrospinal fluid abnormalities, showing an inflammatory process with elevated protein, lymphocytic pleocytosis, and IgG synthesis are common in the early stages. MRI of the brain is often normal in the early stages, with later neuroimaging showing diffuse cerebellar atrophy. Because of the board differential diagnosis associated with PNS, an extensive diagnostic workup is necessary to establish the diagnosis of potentially treatable underlying tumors [7]. In $60 \%$ of cases, paraneoplastic cerebellar degeneration is associated with the 30 distinct onco-neuronal antibodies [13]. An association between specific antibodies, cancer 
type, and PNS has been reported [14]. Notably, anti-Hu are associated with small-cell lung cancer, anti-Tr/DNER with Hodgkin lymphoma, and anti-Yo with ovarian and breast cancer. These antibodies have great diagnostic as well as some prognostic value. Anti-Yo antibodies, also called Purkinje cell cytoplasmic antibodies type 1 (PCA-1), recognize 3 Purkinje cell intracellular antigens, the cerebellar degeneration-related protein 2 (CDR2), the cerebellar degeneration-related protein 3 (CDR3), and the cerebellar degeneration-related protein 1 (CDR1). They result in either extensive Purkinje cell loss and less extensive granule cell loss, leading to cerebellar dysfunction, or intense cell differentiation, rather than cell death, leading to changes of the neurons' function $[1,10]$.

In addition to autoantibodies, cytotoxic T cells seem to be important in the pathogenesis of paraneoplastic cerebellar degeneration. According to Tanaka et al. [15], human leukocyte antigen (HLA) class II molecules, which are present on the surface of antigen-presenting cells, activate the antibody production by $\mathrm{B}$ cells, through $\mathrm{T}$ cell activation. As $\mathrm{T}$ cells are necessary for the anti-Yo antibodies production, HLA class II molecules are considered to participate in the production of anti-Yo antibodies.

As all PNS, paraneoplastic cerebellar degeneration has no single effective treatment. Given the immune-mediated pathogenesis, concomitant immunotherapy, such as plasma exchange, corticosteroids, and immunoglobulin injections, is often prescribed but has only limited activity. Oncological treatments can provide various levels of relieve or stabilization of the symptoms [11]. Rarely, dramatic improvements were reported. Thus, although PNS can have a favorable effect on the oncological prognosis of OC patients, the residual morbidity of these patients due to their neurological complications is impeding their quality of life.

This case study confirms the observation that optimal oncological management of the underlying malignancy provides the best chances for complete neurological recovery and that the presence of PNS can be associated with better oncological outcomes.

\section{Statement of Ethics}

Our patient provided written informed consent, permitting us to disclose details relating to her case.

\section{Conflict of Interest Statement}

There are no conflicts of interest to declare.

\section{Funding Sources}

The authors did not receive any funding.

\section{Author Contributions}

A. Liapi: data collection, literature review, and writing of the original draft; A. Sarivalasis: conceptualization, writing of the review, and editing. Both authors read and approved the final version of the manuscript.

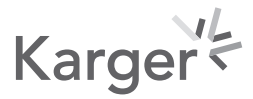




\section{Case Reports in Oncology}

\begin{tabular}{l|l}
\hline Case Rep Oncol 2020;13:1006-1012 \\
\hline DOI: 10.1159/000509029 & $\begin{array}{l}\text { @ } 2020 \text { The Author(s). Published by S. Karger AG, Basel } \\
\text { www.karger.com/cro }\end{array}$ \\
\hline
\end{tabular}

Liapi/Sarivalasis: Paraneoplastic Neurological Syndrome Combined with Ovarian Cancer

\section{References}

1 Zaborowski MP, Spaczynski M, Nowak-Markwitz E, Michalak S. Paraneoplastic neurological syndromes associated with ovarian tumors. J Cancer Res Clin Oncol. 2015;141(1):99-108.

2 Darnell RB, Posner JB. Paraneoplastic syndromes involving the nervous system. N Engl J Med. 2003;349(16): 1543-54.

3 du Bois A, Reuss A, Pujade-Lauraine E, Harter P, Ray-Coquard I, Pfisterer J. Role of surgical outcome as prognostic factor in advanced epithelial ovarian cancer: a combined exploratory analysis of 3 prospectively randomized phase 3 multicenter trials: by the Arbeitsgemeinschaft Gynaekologische Onkologie Studiengruppe Ovarialkarzinom (AGO-OVAR) and the Groupe d'Investigateurs Nationaux Pour les Etudes des Cancers de l'Ovaire (GINECO). Cancer. 2009;115(6):1234-44.

4 Bristow RE, Tomacruz RS, Armstrong DK, Trimble EL, Montz FJ. Survival effect of maximal cytoreductive surgery for advanced ovarian carcinoma during the platinum era: a meta-analysis. J Clin Oncol. 2002;20(5): 1248-59.

5 Vergote I, Tropé CG, Amant F, Kristensen GB, Ehlen T, Johnson N, et al. Neoadjuvant chemotherapy or primary surgery in stage IIIC or IV ovarian cancer. N Engl J Med. 2010;363(10):943-53.

6 Diener HC, Dichgans J. Pathophysiology of cerebellar ataxia. Mov Disord. 1992;7(2):95-109.

7 Hadjivassiliou M, Martindale J, Shanmugarajah P, Grünewald RA, Sarrigiannis PG, Beauchamp N, et al. Causes of progressive cerebellar ataxia: prospective evaluation of 1500 patients. J Neurol Neurosurg Psychiatry. 2017;88(4):301-9.

8 Shalowitz DI, Epstein AJ, Ko EM, Giuntoli RL. Non-surgical management of ovarian cancer: prevalence and implications. Gynecol Oncol. 2016;142(1):30-7.

9 Dalmau J, Rosenfeld MR. Paraneoplastic syndromes of the CNS. Lancet Neurol. 2008;7(4):327-40.

10 Giometto B, Grisold W, Vitaliani R, Graus F, Honnorat J, Bertolini G, et al. Paraneoplastic neurologic syndrome in the PNS Euronetwork database: a European study from 20 centers. Arch Neurol. 2010;67(3):330-5.

11 Pelosof LC, Gerber DE. Paraneoplastic syndromes: an approach to diagnosis and treatment. Mayo Clin Proc. 2010;85(9):838-54.

12 Graus F, Delattre JY, Antoine JC, Dalmau J, Giometto B, Grisold W, et al. Recommended diagnostic criteria for paraneoplastic neurological syndromes. J Neurol Neurosurg Psychiatry. 2004;75(8):1135-40.

13 Jarius S, Wildemann B. 'Medusa head ataxia': the expanding spectrum of Purkinje cell antibodies in autoimmune cerebellar ataxia. Part 2: Anti-PKC-gamma, anti-GluR-delta2, anti-Ca/ARHGAP26 and anti-VGCC. J Neuroinflammation. 2015;12(1):167.

14 Afzal S, Recio M, Shamim S. Paraneoplastic cerebellar ataxia and the paraneoplastic syndromes. Proc (Bayl Univ Med Cent). 2015;28(2):217-20.

15 Tanaka M, Tanaka K, Tokiguchi S, Shinozawa K, Tsuji S. Cytotoxic T cells against a peptide of Yo protein in patients with paraneoplastic cerebellar degeneration and anti-Yo antibody. J Neurol Sci. 1999;168(1):28-31. 